\title{
Efectos de un programa de educación para la seguridad en el deporte en escolares de secundaria

\author{
Effects of an education program for safety in sport in secondary school students
}

\author{
Pedro Ángel Latorre Román, José Carlos Cámara Pérez, Antonio Pantoja Vallejo
}

\author{
Universidad de Jaén
}

\begin{abstract}
Resumen: El objetivo del estudio es analizar los efectos de la implantación de un Programa de Educación para la Seguridad en las actividades físicodeportivas escolares (ESAFE) sobre los factores psicológicos y de personalidad relacionados con el accidente deportivo en escolares de Educación Secundaria. Los participantes de este estudio son escolares de Educación Secundaria de un IES de la provincia de Jaén. El total de sujetos fue de 149 alumnos (Edad= 13.62 años, DT= 1.22). Mediante un ensayo controlado fueron distribuidos en un grupo control (73 participantes, Edad= 13.60 años, $\mathrm{DT}=1.22$ ) y otro experimental (76 participantes, Edad= 13.64 años, $\mathrm{DT}=1.24$ ). Las variables analizadas han sido la búsqueda de sensaciones, la percepción del riesgo, propensión al accidente y por otro lado el número de lesiones y accidentes. Los resultados muestran que tras la intervención, las diferencias postest-pretest son significativamente mayores $(\mathrm{p}<0.001)$ en seguridad pasiva y activa y en la escala total de seguridad en el grupo experimental. El programa ESAFE ha sido capaz de reducir de manera significativa $(\mathrm{p}<0.05)$ la búsqueda de sensaciones en el deporte. El programa ESAFE se puede emplear como un recurso educativo en la sensibilización sobre los riesgos presentes en las actividades físico-deportivas escolares y por lo tanto en la educación de la seguridad. Sería interesante su incorporación en la práctica de los docentes de Educación Física, tanto en la evaluación inicial como durante el proceso de enseñanza y aprendizaje.
\end{abstract}

Palabras clave: educación, seguridad, deporte escolar, búsqueda de sensaciones, percepción del riesgo.

\begin{abstract}
The purpose of the study is to analyze the effects of the implementation of an Education Program for Safety in physical and sport activities on psychological and personality factors related to accidents as a result of sport of Secondary Education students. Participants in this study were Secondary School students, students who were in 1st and 3rd year from a Secondary School in the province of Jaen. The total number of participants were 149 students (age $=13.62$ years, $\mathrm{SD}=1.22$ ). By means of a controlled trial they were divided into a control group (73 participants, age $=13.60$ years, $\mathrm{SD}=1.22)$ and into an intervention group (76 participants, age $=13.64$ years, $\mathrm{SD}=1.24)$. The variables analyzed were sensation seeking, risk perception, propensity of accident and the number of injuries and accidents. Results show that after the intervention of the ESAFE program, pretestposttest differences are significantly higher $(p<0.001)$ in the two scales of safety and in the full scale of the intervention group. The ESAFE program has been able to reduce significantly $(\mathrm{p}<0.05)$ sensation seeking in sport. In conclusion, the ESAFE program can be used as an educational resource in raising awareness of the risks involved in physical and sports activities in school and, therefore, in safety education. So it would be interesting to incorporate it into the practice of PE teachers both in the initial evaluation and during the whole process of teaching and learning.
\end{abstract}

Keywords: education, safety, school sport, sensation seeking, risk perception.

\section{Introducción}

La práctica deportiva conlleva indudables beneficios para la salud, sin embargo, supone en algunos casos un riesgo de lesiones y accidentes. Latorre et al. (2012), Latorre et al., (2007) y Morillas (2003) señalan riesgos importantes en las instalaciones deportivas de los centros educativos y en determinados planteamientos didácticos en Educación Física(EF). Debido a la importancia que este problema representa en el contexto deportivo, diversos estudios han tratado de identificar los factores que pueden incidir en el riesgo de los deportistas a lesionarse. Determinados aspectos psicológicos y de personalidad como el estrés, la ansiedad competitiva, la impulsividad, las motivaciones de logro, el locus de control, la búsqueda de sensaciones, la competencia percibida, el autocontrol, los recursos de afrontamiento y la percepción del riesgo (Abenza, Olmedilla, Ortega \& Esparza, 2009; Greening, Stoppelbein, Chandler \& Elkin 2005; Ivarsson \& Urban, 2010) pueden establecer que un sujeto sea más propenso a conductas de riesgo.

Las lesiones no intencionales son un importante problema de salud infantil cuando los niños deben asumir los riesgos durante el juego, todo ello, basándose en la percepción que se tenga de éstos. Por tanto, uno de los elementos más importantes en el estudio de la accidentalidad infantil hace referencia a la percepción del riesgo, llegando a incluirse en los modelos de conducta saludable (Van der Pligt, 1994).

La percepción del riesgo está afectada por factores internos relacionados con el sexo, la edad, las experiencias previas, la competencia

Fecha recepción: 01-12-12 - Fecha envío revisores: 02-12-12 - Fecha de aceptación: 29-01-13 Pedro Latorre Román

Campus de las lagunillas (Edifico D2)

23071, Jaén

platorre@ujaen.es percibida y la personalidad. En un análisis de diversos autores, Lópezy Osca (2007) indican que los jóvenes se caracterizan por subestimar la posibilidad de sufrir determinados riesgos, no los perciben de una forma holística, los detectan más lentamente y tienden a sobreestimar sus destrezas. Para Kontos (2004) altos niveles de toma de riesgos, bajos niveles de riesgo percibido y bajos niveles de estimación de habilidad, son factores de riesgo de lesiones. Además, las niñas reportan mayores niveles de riesgo percibido y niveles más bajos de asunción de riesgos que los varones. En este sentido, Hillier y Gorronguiello (1998) comprobaron que los niños valoraban más bajo el riesgo que las chicas y que los escolares de 6 años de edad observaban menos riesgos y más lentamente que los de 10 años. DiLillo, Potss y Himes (1998) y Peterson, Brazeal, Olivery Bull (1997) sugieren que con el aumento de la edad, los niños perciben las situaciones como menos peligrosas debido al proceso de familiarización con los riesgos. Schwebel y Barton (2005) indican que los niños con más frecuencia atribuyen sus lesiones a la mala suerte, lo que les lleva a experimentar lesiones repetitivas, mientras que las niñas atribuyen las lesiones a sus propios comportamientos, haciendo que alteren su conducta en situaciones futuras para evitar lesiones. Por tanto, los niños participan en actividades de mayor riesgo físico que las niñas (Morrongiello \& Rennie, 1998). Además, socialmente, los chicos perciben menos riesgo en los deportes, de acuerdo con el estereotipo masculino imperante para éstos. En cambio, para las niñas, su estereotipo deportivo está más alejado de la agresividad, contacto físico y la asunción de riesgos que se consideran necesarios en los deportes de contacto como el fútbol. Por tanto, desde un punto de vista psicosocial, a las niñas se les enseña a desconfiar de los riesgos y a los niños a aceptarlos (Harris \& Miller, 2000). Por otro lado, la falta de experiencia y no preocuparse por las consecuencias graves, pueden desensibilizar a los niños sobre los riesgos potenciales para su salud (Greening et al., 2005). En este sentido, DiLillo et al., (1998) y Horvath y Zuckerman 
(1993) han sugerido que las experiencias previas de lesión pueden tener un efecto desensibilizante en los niños, lo que reduce las estimaciones de riesgo en el futuro. Los sujetos que no han sufrido consecuencias negativas como resultado de su comportamiento arriesgado, mostrarían un menor riesgo en sus sentencias o una desensibilización al riesgo en el futuro (Greening et al., 2005). Desde la perspectiva de la teoría socialcognitiva (Bandura, 1986), la percepción del riesgo de lesión debe ser mayor como consecuencia de haber experimentado las consecuencias negativas en el pasado, por lo tanto, reduciendo la probabilidad de conductas de riesgo de repetición.

Teniendo en cuenta los factores de personalidad, la búsqueda de sensaciones, está muy relacionada con la accidentalidad y la percepción del riesgo. Zuckerman (1979) considera que la búsqueda de sensaciones supone una necesidad de experimentar variadas y complejas sensaciones y el deseo de correr riesgos físicos y sociales por el simple deseo de disfrutar de tales experiencias. El alto buscador de sensaciones se caracteriza por su tendencia a hacer aquellas cosas que un bajo buscador de sensaciones consideraría peligrosas y arriesgadas, la diferencia entre unos y otros es la distinta valoración que se hace del riesgo. Los hombres experimentan mayor búsqueda de sensaciones que las mujeres y puede ser debido tanto a factores biológicos como a factores de socialización; en cuanto a la edad parece existir una relación negativa entre edad y búsqueda de sensaciones (Chico, 2000). Por otro lado, es muy importante tener en cuenta otro factor de personalidad que afecta a la percepción del riesgo como es el egocentrismo de los adolescentes, entendido éste como la tendencia de éstos a pensar que ellos son únicos, por lo que no pueden ser vulnerables a los riesgos para la salud como la persona media típica (Elkind, 1967). El egocentrismo en relación con la percepción del riesgo refleja un sentimiento de inmortalidad. Sin embargo, Greening et al., (2005) indican que un aumento del egocentrismo no predice los cambios en la percepción del riesgo. En este sentido, Dolcini et al. (1989) revelan una pequeña relación positiva entre el egocentrismo adolescente y el riesgo percibido de daño, siendo la relación significativa sólo para las niñas.

La forma en que los sujetos perciben el riesgo en las actividades deportivas escolares es un factor relevante en la explicación de cómo en esas situaciones se desenvuelven y un tópico a considerar en la seguridad de la clase de EF. La educación para la seguridad en el deporte debe aspirar a que los niños sepan identificar las fuentes de riesgo, que adquieran una percepción ajustada del nivel de riesgo asociado a éste y que desarrollen estrategias para evitar dichos riesgos. Parte de estas consideraciones, son una exigencia curricular en España en el ámbito de la Educación Primaria (RD 1513/2006, de 7 de diciembre, por el que se establecen las enseñanzas mínimas de la Educación Primaria, BOE 812-2006). En esta tarea educativa es importante contar con instrumentos para poder evaluar la percepción del riesgo asociado al deporte escolar. Instrumentos de estas características son esenciales para la satisfacción de diversos objetivos, como son: determinar grupos y personas de riesgo que puedan presentar una percepción del riesgo distorsionada, establecer la línea base desde la que iniciar una intervención eidentificar problemas específicos de percepción del riesgo asociados a determinados aspectos (seguridad pasiva de materiales, equipamientos e instalaciones y seguridad activa en relación a los planteamientos prácticos por parte del profesor). Y por último, evaluar la eficacia de una estrategia de intervención.

A nuestro conocimiento, existen escasos estudios sobre la incorporación de programas educativos de Educación para la Seguridad en el deporte escolar, destacándose el programa «Aprende a Crecer con Seguridad» desarrollado por la Junta de Andalucía y determinados manuales como el «Manual de Seguridad de los Centros Educativos (2002)» de la Junta de Andalucía, en el que se hace mención especial a las actividades en la clase de EF.

El objetivo del estudio es analizar los efectos de la implantación de un programa de educación para la seguridad en las actividades físicodeportivas escolares (ESAFE) sobre los factores psicológicos y de personalidad relacionados con el accidente deportivo en escolares de Educación Secundaria.

\section{Método}

\section{Participantes}

Los participantes de este estudio son escolares de Educación Secundaria Obligatoria (ESO), alumnos y alumnas que cursaban $1^{\circ}$ y $3^{\circ}$ de ESO en un IES de la provincia de Jaén. El total de sujetos fue de 149 alumnos (Edad=13.62 años, DT=1.22). En un ensayo controlado, los sujetos fueron asignados en dos grupos equivalentes por sexo y grupo educativo: grupo control (73 participantes, Edad= 13.60 años, DT= 1.22) y grupo experimental (76 participantes, $E d a d=13.64$ años, DT= 1.24).

\section{Instrumentos de recogida de datos}

Se han utilizado tres cuestionarios relacionados con la percepción del riesgo en actividades físico-deportivas escolares, la propensión al accidente deportivo y con la búsqueda de sensaciones para niños y adolescentes. A continuación, se detallan las características principales de cada uno de los cuestionarios utilizados. La «Escala de percepción del riesgo en actividades físico-deportivas escolares» (Cámara, 2012), está basada en el planteamiento de reactivos que evocan una valoración del riesgo percibido ante una serie de situaciones de actividad físicodeportiva en la escuela. El cuestionario está formado por 16 ítems agrupados en dos subescalas con 8 ítems cada una: seguridad pasiva (relacionada con la seguridad de los espacios y equipamientos deportivos) y seguridad activa (relacionada con la seguridad planteada en la realización de ejercicios y propuestas lúdicas en la clase de EF). La valoración del riesgo se establece en una escala graduada de respuesta («1=Nada de peligro» hasta «5=Muy, muy peligroso»). La puntación total de la escala es de 80 puntos posibles como máximo, que indicaría alta percepción del riesgo y 16 como mínimo o baja percepción del riesgo. La «Escala de propensión al accidente deportivo en escolares (EPAD)» adopta el formato de autoinforme y está compuesto por 27 ítems y formado por 5 subescalas: competencia percibida (CP), búsqueda de sensaciones (BS), competitividad (COMP), asunción de riesgos (AR) y percepción de riesgos (PR) (Latorre, Cámara y Pantoja, 2012). Finalmente, la «Escala de búsqueda de sensaciones para niños y adolescentes(EBS-J)» de Pérez, Ortet, Plá y Simó(1987), mide el rasgo de búsqueda de sensaciones en niños y adolescentes, consta de 50 ítems en una escala dicotómica y se divide en cuatro dimensiones: búsqueda de emociones (BEM), búsqueda de excitación (BEX), desinhibición (DES) y susceptibilidad al aburrimiento (SAB) y una valoración global de la escala(Total escala EBS-J).

\section{Procedimiento}

De manera autoadministrada se cumplimentaron los cuestionarios (pre-test y post-test), atendiéndose en todo momento a las dudas surgidas y garantizando la confidencialidad y el anonimato de las respuestas. La duración de la administración de cada cuestionario fue de 15 a 25 minutos aproximadamente. La recogida de datos se realizó a lo largo del curso escolar 2010-2011. El estudio fue aprobado por el Comité de Ética de la Universidad de Jaén. Se contó con la autorización por parte de la dirección del IES, así como un consentimiento informado por parte de los padres de los alumnos implicados en el estudio.

En cuanto al programa ESAFE, tuvo 5 meses de duración (desde febrero hasta junio) con un conjunto de sesiones que fueron aplicadas a modo de rutinas en un momento concreto de la clase de EF y otras de ellas a lo largo del proceso de aprendizaje en sesiones monográficas. Diariamente, en cada sesión práctica de EF, los alumnos (por grupos de 3 y rotando durante todo el proceso del programa) valoraban el estado de la instalación donde se iba a realizar la sesión (porterías, suelo, ganchos, anclajes, redes...) basándose en el modelo observacional de la seguridad de las instalaciones deportivas de Latorre y Muñoz (2011). Además, realizaban el calentamiento previo a la actividad y la vuelta a la calma con el objetivo de concienciar al alumnado de su importancia para la prevención de lesiones. Igualmente, al finalizar cada una de las sesiones, otros 3 alumnos diferentes y rotando durante todo el proceso del programa, rellenaron una hoja de observación sobre aspectos de per- 
cepción del riesgo de la sesión práctica que se acababa de realizar. A su vez, se contabilizaron el número de lesiones y accidentes sufridos por el alumnado en sus actividades deportivas escolares y extraescolares a lo largo de 5 meses. Los contenidos de las sesiones monográficas están relacionados con la concienciación, sensibilización y percepción del riesgo en las actividades físico-deportivas escolares. A continuación se detallan los contenidos principales de estas sesiones monográficas:

- Visualización de fotografías de instalaciones deportivas con elementos de riesgo.

- Visualización de vídeos de caídas en actividades físico-deportivas. - Conferencia educativa de «primeros auxilios» (enfermera del SAS).

- Análisis de juegos peligrosos en EF y valoración del riesgo (Latorre et al., 2007).

- Análisis de ejercicios contraindicados (López Miñarro, 2000) tanto en el deporte como en la vida cotidiana.

- Realización de un cómic de accidente deportivo.

- Normas básicas de higiene postural: transporte de mochilas y material deportivo.

- Inspección visual de instalaciones deportivas del centro escolar y del municipio (Modelo observacional de Latorre y Muñoz, 2011)

\section{Análisis estadístico}

Los datos de este estudio se han hallado mediante el programa estadístico SPSS., v.18.0 para Windows, (SPSS Inc, Chicago, USA). Para el análisis de datos se ha utilizado la estadística descriptiva (medias, DT), ANCOVA con el sexo y la edad como covariables para analizar las diferencias de los grupos en el pretest. ANOVA de medidas repetidas para analizar el efecto del programa de intervención ESAFE, ANCOVA empleando como covariables la edad, el sexo y el pretest para analizar las diferencias post-pre entre grupos y prueba t para muestras independientes para analizar las diferencias en el número de lesiones. El nivel de significación se estableció en $\mathrm{p}<0.05$.

\section{Resultados}

De los participantes seleccionados para este estudio todos completaron el programa. No se encuentran diferencias significativas $(\mathrm{p}<0.05)$ en el pretest en ninguna de las variables de estudio. En la tabla 1 podemos observar que existe una reducción significativa $(\mathrm{p}<0.05)$ en la BS después de la intervención en el grupo experimental Además, se muestra un incremento significativo $(\mathrm{p}<0.05)$ de la percepción del riesgo tanto en la componente de seguridad pasiva, activa y escala total de percepción del riesgo, en el grupo control y experimental.

\begin{tabular}{|c|c|c|c|c|c|c|}
\hline Variable & Grupo & $\begin{array}{c}\text { Pre } \\
\text { Media(DT) }\end{array}$ & $\begin{array}{c}\text { Post } \\
\text { Media(DT) }\end{array}$ & p grupo & p tiempo & p interacción \\
\hline BEM & $\begin{array}{l}\text { Control } \\
\text { Experimental }\end{array}$ & $\begin{array}{l}4.52(3.40) \\
5.53(2.99)\end{array}$ & $\begin{array}{l}4.97(3.31) \\
5.71(3.15)\end{array}$ & NS & NS & NS \\
\hline BEX & $\begin{array}{l}\text { Control } \\
\text { Experimental }\end{array}$ & $\begin{array}{l}4.10(1.71) \\
3.93(2.02)\end{array}$ & $\begin{array}{l}4.14(1.81) \\
4.32(2.14)\end{array}$ & NS & NS & NS \\
\hline DES & $\begin{array}{l}\text { Control } \\
\text { Experimental }\end{array}$ & $\begin{array}{l}3.64(2.38) \\
3.30(2.48) \\
\end{array}$ & $\begin{array}{l}3.99(2.76) \\
3.53(2.67) \\
\end{array}$ & NS & NS & NS \\
\hline $\mathrm{SAB}$ & $\begin{array}{l}\text { Control } \\
\text { Experimental }\end{array}$ & $\begin{array}{l}4.07(2.55) \\
4.13(2.39) \\
\end{array}$ & $\begin{array}{l}.93(2.48) \\
3.89(2.35) \\
\end{array}$ & NS & NS & NS \\
\hline $\begin{array}{l}\text { Total escala } \\
\text { EBS-J }\end{array}$ & $\begin{array}{l}\text { Control } \\
\text { Experimental }\end{array}$ & $\begin{array}{l}16.32(6.16) \\
16.89(6.52)\end{array}$ & $\begin{array}{l}17.02(6.54) \\
17.44(7.40)\end{array}$ & NS & NS & s \\
\hline $\mathrm{CP}$ & $\begin{array}{l}\text { Control } \\
\text { Experimental }\end{array}$ & $\begin{array}{l}27.36(8.93) \\
24.64(8.78)\end{array}$ & $\begin{array}{l}25.23(9.95) \\
24.08(9.28)\end{array}$ & NS & 0.017 & NS \\
\hline BS & $\begin{array}{l}\text { Control } \\
\text { Experimental }\end{array}$ & $\begin{array}{l}20.16 \text { (8.58) } \\
20.54(8.59) \\
\end{array}$ & $\begin{array}{l}21.19(8.39) \\
19.05(8.57)^{*}\end{array}$ & NS & NS & 0.018 \\
\hline COMP & $\begin{array}{l}\text { Control } \\
\text { Experimental }\end{array}$ & $\begin{array}{l}13.60(5.35) \\
13.54(5.40)\end{array}$ & $\begin{array}{l}14.30(5.38) \\
13.16(5.43)\end{array}$ & NS & NS & NS \\
\hline AR & $\begin{array}{l}\text { Control } \\
\text { Experimental }\end{array}$ & $\begin{array}{l}15.53(5.56) \\
14.64(5.26)\end{array}$ & $\begin{array}{l}13.70(5.30) \\
13.32(5.55)\end{array}$ & NS & $<0.001$ & NS \\
\hline PR & $\begin{array}{l}\text { Control } \\
\text { Experimental }\end{array}$ & $\begin{array}{l}16.92(4.88) \\
15.88(5.55)\end{array}$ & $\begin{array}{l}15.29(4.80) \\
15.84(5.16)\end{array}$ & NS & NS & NS \\
\hline SEG PAS & $\begin{array}{l}\text { Control } \\
\text { Experimental }\end{array}$ & $\begin{array}{l}19.49(3.94) \\
19.16(5.02)\end{array}$ & $\begin{array}{l}21.24(5.19)^{* *} \\
35.09(2.74)^{* *}\end{array}$ & $<0.001$ & $<0.001$ & $<0.001$ \\
\hline SEG ACT & $\begin{array}{l}\text { Control } \\
\text { Experimental }\end{array}$ & $\begin{array}{l}16.63(4.71) \\
18.27(4.96)\end{array}$ & $\begin{array}{l}18.28(4.77)^{* *} \\
34.21(3.46)^{* *}\end{array}$ & $<0.001$ & $<0.001$ & $<0.001$ \\
\hline SEG TOT & $\begin{array}{l}\text { Control } \\
\text { Experimental }\end{array}$ & $\begin{array}{l}36.12(7.31) \\
37.43(9.26)\end{array}$ & $\begin{array}{l}39.53(8.65)^{* *} \\
69.30(5.61)^{* *}\end{array}$ & $<0.001$ & $<0.001$ & $<0.001$ \\
\hline
\end{tabular}

En la tabla 2 se exponen los resultados de las diferencias entre el postest y el pretest por grupos incluyendo como covariables la edad, el sexo y el pretest. Se observa que el grupo experimental ha obtenido una mejora significativa $(\mathrm{p}<0.001)$ en seguridad pasiva, activa y en la escala total de seguridad en relación con el grupo control.

En relación con el número de lesiones y accidentes recogidos en cinco meses de intervención, se encontraron diferencias significativas $(\mathrm{p}=0.037)$ entre el grupo experimental (Media $=0.49, \mathrm{DT}=0.60)$ y el grupo control (Media=0.73, DT=0.76).

\begin{tabular}{cccc}
\multicolumn{4}{l}{ Tabla 2. Diferencias pos tes t-p retest por grupos tras la intervención. } \\
\hline & Grupo control & Grupo experimental & $p$-valor \\
\hline SEG PAS & $1.75(6.75)$ & $15.93(5.26)$ & $\mathrm{p}<0.001$ \\
SEG ACT & $1.65(6.59)$ & $15.94(6.08)$ & $\mathrm{p}<0.001$ \\
SEG TOTAL & $3.41(12.10)$ & $31.87(10.33)$ & $\mathrm{p}<0.001$ \\
\hline Seguridad pasiva (SEG PAS), seguridad activa (SEG ACT)y escala total seguridad (SEG TOT)
\end{tabular}

\section{Discusión}

El programa ESAFE tiene como objetivo concienciar al alumnado de los peligros y situaciones de riesgo que se pueden desencadenar en cualquier sesión de EF en el entorno escolar. Los hallazgos más importantes de este estudio han sido que tras la intervención del programa ESAFE ha resultado un incremento significativo $(\mathrm{p}<0.001)$ en la percepción del riesgo en todos sus factores, seguridad pasiva, seguridad activa y en la escala de seguridad total. Estos resultados se han observado tanto en el grupo control como en el grupo experimental, sin embargo, las diferencias postest-pretest por grupos son significativamente mayores ( $\mathrm{p}<0.001$ ) en la seguridad pasiva, activa y en la escala total de seguridad en el grupo experimental. Puede ser que las mejoras en la percepción del riesgo del grupo control hayan sido influenciadas por una información cruzada entre compañeros de diferentes clases, aunque se controló este factor (práctica de las sesiones en espacios cerrados e imposibilidad de llevarse los documentos físicos de apuntes fuera del aula) siempre es posible que existiera una comunicación entreel alumnado.

Por otra parte, la intervención del programa ESAFE no ha influido en la modificación de los factores psicológicos y de personalidad relacionados con conductas de riesgo, en este caso con la propensión al accidente y la escala EBS-J. Creemos que esto puede ser debido a que estos aspectos psicológicos son elementos evolutivos ineludibles en la adolescencia y difíciles de modificar. En este sentido, Oliva (2007) señala que la corteza prefrontal, estructura fundamental en muchos procesos cognitivos experimenta un importante desarrollo a partir de la pubertad que no culmina hasta los primeros años de la adultez temprana. Otros cambios afectan al circuito mesolímbico, relacionado con la motivación y la búsqueda de recompensas, que va a verse influido por las alteraciones hormonales asociadas a la pubertad. Como consecuencia de esas modificaciones, durante los primeros años de la adolescencia se produce un cierto desequilibrio entre ambos circuitos cerebrales, el cognitivo y el motivacional, que puede generar cierta vulnerabilidad y justificar el aumento de la impulsividad y las conductas de asunción de riesgos durante la adolescencia. A su vez, Sánchez y Cantón (2001) admiten que se han realizado muchas investigaciones con el objetivo de conocer qué motiva a las personas para realizar conductas que implican un riesgo físico, que pueden causar graves daños e incluso la muerte. Algunas de ellas han explorado la posibilidad de que la variable de búsqueda de sensaciones sea la dimensión que oriente a las personas a realizar este tipo de actividades. Asimismo, aseguran que las personas que practican actividades que puedan suponer un riesgo soportan, en ocasiones, cierto nivel de dolor y ponen de manifiesto la necesidad de demostrar competencia. Esta necesidad de aprobación social es una característica común al período adolescente que aumenta el ego de la persona que acepta el reto de la situación de riesgo, y esto es muy difícil de modificar. En todo caso, el programa ESAFE ha sido capaz de reducir de manera significativa $(\mathrm{p}<0.05)$ la BS de la escala de propensión al accidente en el grupo experimental.

Otro hallazgo relevante de este estudio es el menor número de lesiones y accidentes deportivos en el grupo experimental, aunque no se ha podido precisar su relación con el incremento en la percepción del 
riesgo y la reducción de la BS. En este sentido, Munugarren (2005) señala que es importante que los niños aprendan hábitos conductuales que les permitan en el futuro desarrollar estrategias para su seguridad y promover así estilos de vida saludables. Al respecto la Junta deAndalucía ha puesto en marcha el programa «Aprendea Crecer con Seguridad». El principal objetivo de este programa es concienciar sobre los accidentes a niños, niñas, padres, madres y profesorado, mediante una apuesta decidida por la implantación de una «Cultura de la Prevención» en el ámbito escolar, el hogar y el trabajo. Este objetivo permitirá reducir el número de accidentes infantiles y, en un futuro, la reducción de accidentes y enfermedades de carácter laboral. En este sentido se plantea la seguridad infantil como paso previo a la seguridad en el trabajo. Sin embargo, este programa no aborda aspectos específicos de seguridad en la clase de EF, que es el escenario escolar de mayor riesgo de accidentes. Debido a la inexistencia de estudios semejantes sobre los efectos de un programa de educación para la seguridad en el deporte, no hemos podido realizar una discusión comparada con otros autores.

Por último, el programa ESAFE presenta una relación importante con el curriculum escolar. En la tabla 3 relacionamos el programa ESAFE con las competencias básicas, bloques de contenidos y objetivos de etapa (ESO) y área (EF).

\begin{tabular}{|c|c|c|c|}
\hline $\begin{array}{l}\text { Competencias } \\
\text { Básicas }\end{array}$ & $\begin{array}{l}\text { Bloque de } \\
\text { Contenidos }\end{array}$ & $\begin{array}{c}\text { Objetivos } \\
\text { de etapa (ESO) }\end{array}$ & $\begin{array}{l}\text { Objetivos } \\
\text { de Área (EF) }\end{array}$ \\
\hline $\begin{array}{l}\text { Competencia en el conocimiento y la } \\
\text { interacción con el mundo físico y nat ural }\end{array}$ & $\begin{array}{l}\text { Condición Física y } \\
\text { Salud }\end{array}$ & \multirow{4}{*}{ A, C, D, K y L } & \multirow{4}{*}{$\begin{array}{l}1,2,3,4,7,8, \\
10,14,17\end{array}$} \\
\hline Competencia social y ciudadana & \multirow{3}{*}{ Juegos y Deportes } & & \\
\hline Competencia cultural yartística & & & \\
\hline $\begin{array}{l}\text { Competencia para la autonomía e } \\
\text { iniciativa personal }\end{array}$ & & & \\
\hline
\end{tabular}

\section{Limitaciones}

El efecto de información cruzada de los alumnos del centro en ambos grupos ha podido influir en que el grupo control mejore en percepción del riesgo. Por lo tanto, para futuros estudios se podrían determinar diferentes grupos en diferentes centros educativos, de distinta localidad o, incluso, de distintas provincias. Aunque se realizó una asignación aleatoria de los cursos a los grupos control y experimental, la no randomización individual de los participantes en los grupos es una limitación de este estudio.

\section{Conclusiones}

El programa ESAFE produce una mayor percepción del riesgo en las actividades físico-deportivas escolares en los alumnos participantes, a su vez reduce la BS en el deporte y se contabilizan menos lesiones en el grupo que recibe el programa. Por tanto, el ESAFE puede considerarse un programa educativo eficaz para la educación en la seguridad en el deporte escolar y podría incorporarse de manera transversal en la práctica diaria del profesor de EF.

\section{Referencias}

Abenza, L., Olmedilla, A., Ortega, E., \& Esparza, F. (2009). Estados de ánimo y adherencia a la rehabilitación de deportistas lesionados. Apunts Medicina de l'Esport, 161, 29-37.

Bandura, A. (1986). Social foundations of thought and action: A social cognitive theory. Englewood Cliffs, N.J: Prentice Hall.

Cámara, J.C. (2012). Percepción del riesgo y accidentalidad en la clase de Educación Física en alumnado de Educación Secundaria: una propuesta de intervención didáctica (Tesis inédita de doctorado). Universidad de Jaén, Jaén.

Chico, L. (2000). Búsqueda de sensaciones. Psicothema, 12(2), 229-235.

Consejería de Educación y Ciencia. (2002). Manual de seguridad en los centros educativos. Dirección general de construcciones yequipamiento escolar. Sevilla: Junta de Andalucía.

DiLillo, D., Potss, R., \& Himes, S. (1998). Predictors of Children’s RiskAppraisals. Journal of Applied Developmental Psychology, 19(3), 415-427.

Dolcini, M.M., Cohn, L.D., Adler, N.E., Millstein, S.G., Irwin, C.E., Kegeles, S.M\& Stone, G(1989).Adolescent egocentrism and feelings of invulnerability: Are they related? Journal of Early Adolescence, 9(4), 409-418.

Elkind, D. (1967). Egocentrism in adolescence. Child Development, 38, 10251034.
Greening, L., Stoppelbein, L., Chandler, C., \& Elkin, D. (2005). Predictors of Children's and Adolescents' Risk Perception. Journal of Pediatric Psychology, 30(5), 425-435.

Harris, M.B., \& Miller, K.C. (2000). Gender and perceptions of danger. Sex Roles, 43, 843-863.

Hillier, L., \& Morrongiello, B.A.(1998). Age and gender differences in school-age children's appraisal of injury risk. Journal of Pediatric Psychology, 23(4), 229238.

Horvath, P., \& Zuckerman, M. (1993). Sensation seeking and risk appraisal and risk behavior. Personality y Individual Differences, 14, 41-52.

Ivarsson, A., \& Urban, J. (2010). Psychological factors as predictors of injuries among senior soccer players. A prospective study. Journal of Sports Science and Medicine, 9, 347-352.

Kontos, A. (2004). Perceived Risk, Risk Taking, Estimation of Ability and Injury AmongAdolescent Sport Participants. Journal of Pediatric Psychology, 29(6), 447-455.

Latorre, P.A., Mejía, J.A., Gallego, M., Muñoz, A., Santos, M.A, \& Adell, M. (2012).El análisis de la seguridad de las instalaciones deportivas de los juegos deportivos provinciales de Jaén. Journal of Sport and Health Research, 4(1), 57-66.

Latorre, P.A. (2008). Metodología para el análisis y evaluación de la seguridad de los espacios y equipamientos deportivos escolares. Revista Apunts Educación Física y Deportes, 93, 62-70.

Latorre, P.A., Jiménez, My grupo de trabajo (2007). Análisis y evaluación del riesgo de tareas motrices propuestas en la literatura. Revista de Educación Física, 105, 25-39.

Latorre, P.A., Cámara, J.C, \& Pantoja,A. (en prensa). Factores psicoeducativos que determinan la propensión al accidente deportivo en escolares: Diseño y validación de un cuestionario de evaluación. Manuscrito aceptadopara supublicación en Revista Anales de Psicología.

Latorre, P.A., \& Muñoz. A. (2011). Manual de control de la seguridad de los espacios y equipamientos deportivos de uso escolar. Jaén: Diputación de Jaén.

López, B., \& Osca,A. (2007). Factores explicativos de la accidentalidad en jóvenes: Un análisis de la investigación. Revista de Estudios de Juventud, 79, 75-89.

López-Miñarro, P.A. (2000). Ejercicios desaconsejados en la Actividad Física. Detección yalternativas. Barcelona: INDE.

Ministerio de Educación y Ciencia. Real Decreto 1513/2006, de 7 de Diciembre, por el que se establecen las enseñanzas mínimas de la Educación Primaria. BOE número 293 de 8 de diciembre de 2006.

Ministerio de Educación y Ciencia. Real Decreto 1631/2006, de 29 de diciembre, por el que se establecen las enseñanzas mínimas correspondientes a la Educación Secundaria Obligatoria. BOE número 5 de 5 enero 2007.

Morillas., M. (2003). Calidad y seguridad de las instalaciones y el material deportivo en los Centros de Educación Secundaria y Bachillerato de la provincia de Málaga. Málaga: Servicio de Publicaciones de la Universidad de Málaga.

Morrongiello, B.A, \& Rennie, H. (1998). Why do boys engage in more risk taking than girls? The role of attributions, beliefs, and risk appraisals. Journal of Pediatric Psychology, 23(1), 33-43.

Munugarren, R. (2005). Seguridad y Educación. Prevención de accidentes infantiles. Madrid: FUNDADEPS. Ed. Mapfre.

Oliva,A. (2007). Desarrollo cerebral y asunción de riesgos durante la adolescencia. Apuntes de Psicología, 25(3), 239-254

Pérez, J., Ortet, G., Plá, S., \& Simó, S. (1987). Escala de Búsqueda de Sensaciones para Niños yAdolescentes (EBS-J). Evaluación Psicológica, 3, 283-290.

Peterson, L., Brazeal, T., Oliver, K., \& Bull, C. (1997). Gender and developmental patterns of affect, belief, and behaviors in simulated injury events. Journal of Applied Developmental Psychology, 18(4), 531-546.

Roldan, C. (2002). Manual de Seguridad de los Centros Educativos. Sevilla: Consejería de Educación y Ciencia. Dirección General de Construcciones y Equipamiento Escolar.

Sánchez, M.C., \& Cantón E. (2001). La práctica de actividad físico-deportiva de riesgo como herramienta preventiva de conductas desajustadas psicosocialmente. Revista de Psicología del Deporte, 10(2), 225-236.

Schwebel, D., \& Barton, B. (2005). Contributions of Multiple Risk Factors to Child Injury. Journal of Pediatric Psychology, 30(7), 553-561.

Van der Pligt, J. (1994). Risk appraisal and health behavior. In D. R. Rutter y L. Quine(Eds.), Social psychology and health: European perspectives. (pp.131151). Brookfield VT:Avebury.

Zuckerman, M. (1979). Sensation Seeking: Beyond the Optimal Level of Arousal. Hillsdale, NJ: Erlbaum.

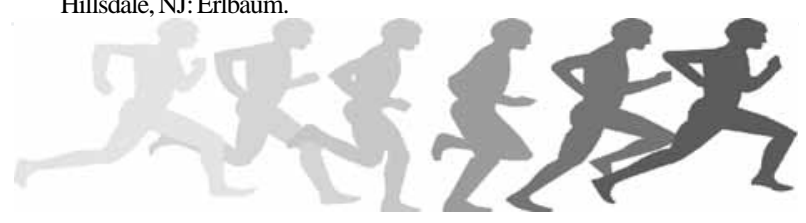

